International Journal of Bifurcation and Chaos, Vol. 12, No. 4 (2002) 815-818

(c) World Scientific Publishing Company

\title{
A NEW SYNCHRONIZATION PRINCIPLE AND APPLICATION TO CHUA'S CIRCUITS
}

\author{
YONGAI ZHENG*, ZENGRONG LIU and JIN ZHOU \\ Department of Mathematics, Shanghai University, \\ Shanghai, 200436, China \\ *Department of Mathematics, Yangzhou University, \\ Yangzhou, 225006, China
}

Received May 15, 2001; Revised June 12, 2001

\begin{abstract}
In the paper we propose a new synchronization principle. To guarantee synchronization between coupled chaotic oscillators, proper coupling constants are selected by the Liapunov stability theory and Hurwitz Theorem. As an example and application, we prove the conjecture [Wu \& Chua, 1994] that synchronization between two chaotic Chua's circuits can be achieved by using the second state as feedback variable for sufficiently large coupling constant.
\end{abstract}

Keywords: Synchronization; Liapunov stability; Lipschitz condition.

\section{Introduction}

Increasing interest has been generated in chaotic synchronization and its practical applications in different fields, particularly in communications [Ogorzalek, 1993; Pecora et al., 1997; Kolumban et al., 1997] since Pecora and Carroll's observation about the possibility of synchronizing two chaotic systems in the so-called drive-response configuration [Pecora \& Carroll, 1990]. In the studies of chaotic synchronization, Chua's circuit plays an important role since it is an extremely simple system and yet exhibits the complex dynamics of bifurcation and chaos. Synchronization of two coupled Chua's circuits has been studied through linear coupling or feedback [Chua et al., 1993; Wu \& Chua, 1994; Chua et al., 1996], among many other approaches. In particular, it was proved in $[\mathrm{Wu} \&$ Chua, 1994] that synchronization of two Chua's circuits with the first state as the feedback variable can be achieved when the coupling constant is large enough. In the same paper, they also conjectured, based on simulations, that synchronization of two Chua's circuits with the second state as the feedback variable can be achieved when the cou- pling constant is large enough. To make synchronization of two Chua's circuits, the second state as the coupling variable is more significant than the first state as the coupling variable from the view of the security of communications. However, rigorous argument to the conjecture has not been given so far to our knowledge. Wang et al. [1999] studied this problem by nonlinear observer design methods. They only found an interval of the coupling constant, in which two Chua's circuits with coupled second variable were indeed synchronized, but they did not exactly find a universal threshold $c^{*}>0$ such that two Chua's circuits with coupled second variable were synchronized for any coupling constant $c>c^{*}$. In this paper, we present a new principle of two coupled chaotic oscillators to guarantee synchronization by using the Liapunov stability method and Hurwitz theorem. As an example and application of the new principle, we prove that the above conjecture is right.

This paper is organized as follows. In Sec. 2 we present a new synchronization principle. In Sec. 3 we apply the new synchronization principle to coupled Chua's circuits. In Sec. 4, we give some concluding remarks. 


\section{A New Synchronization Principle}

Consider two coupled chaotic systems described by

$$
\dot{x}=A x+\phi(x)
$$

and

$$
\dot{\hat{x}}=A \hat{x}+\phi(\hat{x})-c K(\hat{x}-x)
$$

where $x$ and $\hat{x}$ are $n$-dimensional state variables of the master system (1) and the slave system (2) respectively, $c(>0)$ is the coupling constant, and

$$
K=\left(\begin{array}{cccc}
k_{1} & & & \\
& k_{2} & & \\
& & \ddots & \\
& & & k_{n}
\end{array}\right)
$$

where $k_{i}(i=1,2, \ldots, n)$ is constant, $A$ is a $n \times n$ matrix and $\phi(x) \in R^{n}$ is a $n$-dimensional nonlinear continuous function satisfying the Lipschitz condition $\|\phi(x)-\phi(\hat{x})\| \leq \rho\|x-\hat{x}\|$, constant $\rho$ is called the Lipschitz constant, $\|\cdot\|$ denotes the Euclidean norm. With respect to systems 1 and 2, we have the following result:

Theorem 1. Let $E$ be $n \times n$ unit matrix and $\left\{\lambda_{i}\right\}_{i=1}^{n}$ be the eigenvalues of the symmetric matrix $\left((A-c K)+(A-c K)^{T}\right) / 2+\rho E$, if $\max \left\{\lambda_{i}\right\}_{i=1}^{n}<$ 0 , then the master system (1) and the slave system (2) are synchronized in the sense that the synchronization error $e=x-\hat{x}$ tends to zero asymptotically:

$$
\lim _{t \rightarrow \infty} e(t)=0 .
$$

Proof. Consider the error equations

$$
\dot{e}=(A-c K) e+\phi(x)-\phi(\hat{x}) .
$$

Assume that the symmetric matrix $((A-c K)+$ $\left.(A-c K)^{T}\right) / 2+\rho E$ is similar to

$$
\Lambda=\operatorname{diag}\left(\lambda_{1}, \lambda_{2}, \ldots, \lambda_{n}\right)
$$

by a orthogonal matrix $U$, that is $U^{T}[((A-c K)+$ $\left.\left.(A-c K)^{T}\right) / 2+\rho E\right] U=\Lambda$. Let $V(e)=1 / 2\|e\|^{2}$, we will calculate the derivative of $V$ along trajectories of the system (3),

$$
\begin{aligned}
\dot{V} & =\frac{1}{2}(\langle\dot{e}, e\rangle+\langle e, \dot{e}\rangle) \\
& =e^{T} \frac{(A-c K)+(A-c K)^{T}}{2} e+\langle\phi(x)-\phi(\hat{x}), e\rangle \\
& \leq e^{T}\left[\frac{(A-c K)+(A-c K)^{T}}{2}+\rho E\right] e
\end{aligned}
$$

$$
\begin{aligned}
& =e^{T} U \Lambda U^{T} e=\left(U^{T} e\right)^{T} \Lambda\left(U^{T} e\right) \\
& \leq \max \left\{\lambda_{i}\right\}_{i=1}^{n}\left(U^{T} e\right)^{T}\left(U^{T} e\right) \\
& <0 \quad \text { for all } e \neq 0 .
\end{aligned}
$$

Then $V(e) \rightarrow 0$ as $t \rightarrow \infty$, which means that $e \rightarrow 0$ as $t \rightarrow \infty$.

If the form of nonlinear function $\phi(x)$ is taken as

$$
\phi(x)=\left(\varphi\left(x_{1}\right), 0, \ldots, 0\right)
$$

where $\left|\varphi\left(x_{1}\right)-\varphi\left(\hat{x}_{1}\right)\right| \leq \rho\left|x_{1}-\hat{x}_{1}\right|$, we have the following result.

Corollary 1. Let $\left\{\lambda_{i}\right\}_{i=1}^{n}$ be the eigenvalues of the symmetric matrix $\left((A-c K)+(A-c K)^{T}\right) / 2+\rho B$, if $\max \left\{\lambda_{i}\right\}_{i=1}^{n}<0$, then the master system (1) and the slave system (2) are synchronized in the sense specified above, where

$$
B=\left(\begin{array}{llll}
1 & & & \\
& 0 & & \\
& & \ddots & \\
& & & 0
\end{array}\right)
$$

is a $n \times n$ matrix with only a nonzero element 1 .

In order to make synchronization of systems (1) and (2), we only need to choose $c$ such that the eigenvalues of the symmetric matrix $\left((A-c K)+(A-c K)^{T}\right) / 2+\rho E$ $\left(\left((A-c K)+(A-c K)^{T}\right) / 2+\rho B\right)$ are less than zero by Theorem 1 (Corollary 1). Fortunately, it can be finished by the Hurwitz's theorem below.

Theorem 2. (Hurwitz's Theorem) [Rama Mohana Rao, 1980] A necessary and sufficient condition for the negativity of the real parts of all the roots of the polynomial

$$
L(\lambda)=\lambda^{n}+a_{1} \lambda^{n-1}+\cdots+a_{n-1} \lambda+a_{n}
$$

with real coefficients is the positivity of all the principal diagonals of the minors of the Hurwitz matrix,

$$
H_{n}=\left(\begin{array}{ccccccccc}
a_{1} & 1 & 0 & 0 & 0 & 0 & 0 & \cdots & 0 \\
a_{3} & a_{2} & a_{1} & 1 & 0 & 0 & 0 & \cdots & 0 \\
a_{5} & a_{4} & a_{3} & a_{2} & a_{1} & 1 & 0 & \cdots & 0 \\
\vdots & \vdots & \vdots & \vdots & \vdots & \vdots & \vdots & & \vdots \\
0 & 0 & 0 & 0 & 0 & 0 & 0 & \cdots & a_{n}
\end{array}\right)
$$


The Hurwitz theorem is practicable for small $n$. Polynomials of the second, third and fourth degrees are used as examples to illustrate the application of this Theorem:

(i) $\lambda^{2}+a_{1} \lambda+a_{2}$.

The Hurwitz conditions reduce to $a_{1}>0$ and $a_{2}>0$.

(ii) $\lambda^{3}+a_{1} \lambda^{2}+a_{2} \lambda+a_{3}$.

The Hurwitz conditions reduce to $a_{1}>0, a_{2}>0$, $a_{3}>0$ and $a_{1} a_{2}-a_{3}>0$.

(iii) $\lambda^{4}+a_{1} \lambda^{3}+a_{2} \lambda^{2}+a_{3} \lambda+a_{4}$.

The Hurwitz conditions reduce to $a_{1}>0, a_{2}>0$, $a_{3}>0, a_{4}>0$ and $a_{1} a_{2} a_{3}-a_{3}^{2}-a_{1}^{2} a_{4}>0$.

\section{Application to Coupled Chua's Circuits}

The dimensionless form of two Chua's circuits with coupled second variable is described by

$$
\left(\begin{array}{c}
\dot{x}_{1} \\
\dot{x}_{2} \\
\dot{x}_{3}
\end{array}\right)=\left(\begin{array}{c}
\alpha\left(x_{2}-x_{1}+f\left(x_{1}\right)\right) \\
x_{1}-x_{2}+x_{3} \\
-\beta x_{2}-\gamma x_{3}
\end{array}\right)
$$

and

$$
\left(\begin{array}{l}
\dot{\hat{x}}_{1} \\
\dot{\hat{x}}_{2} \\
\dot{\hat{x}}_{3}
\end{array}\right)=\left(\begin{array}{c}
\alpha\left(\hat{x}_{2}-\hat{x}_{1}+f\left(\hat{x}_{1}\right)\right) \\
\hat{x}_{1}-\hat{x}_{2}+\hat{x}_{3}-c\left(\hat{x}_{2}-x_{2}\right) \\
-\beta \hat{x}_{2}-\gamma \hat{x}_{3}
\end{array}\right)
$$

where $f(\cdot)$ is a piecewise-linear function

$$
f\left(x_{1}\right)= \begin{cases}-b x_{1}-a+b & x_{1}>1 \\ -a x_{1} & \left|x_{1}\right| \leq 1 \\ -b x_{1}+a-b & x_{1}<1\end{cases}
$$

where $\alpha>0, \beta>0, \gamma>0$ and $a<b<0$. Using notation defined in Sec. 2, we have

$$
A-c K=\left(\begin{array}{ccc}
-\alpha & \alpha & 0 \\
1 & -1-c & 1 \\
0 & -\beta & -\gamma
\end{array}\right) .
$$

The Lipschitz constant of $f\left(x_{1}\right)$ is $\rho=-a$, i.e.

$$
\left|f\left(x_{1}\right)-f\left(\hat{x_{1}}\right)\right| \leq-a\left|x_{1}-\hat{x}_{1}\right| \text {. }
$$

In this study, the system parameters are chosen to be

$$
\begin{gathered}
\alpha=10.0000, \quad \beta=15.0000, \quad \gamma=0.0385 \\
a=-1.2700, \quad b=-0.6800 .
\end{gathered}
$$

Thus

$$
\begin{aligned}
M & =\frac{(A-c K)+(A-c K)^{T}}{2}-a B \\
& =\left(\begin{array}{ccc}
-8.7300 & 5.5000 & 0.0000 \\
5.5000 & -1.0000-c & -7.0000 \\
0.0000 & -7.0000 & -0.0385
\end{array}\right) \quad(11) \\
|\lambda E-M| & =\left|\begin{array}{ccc}
\lambda+8.7300 & -5.5000 & 0.0000 \\
-5.5000 & \lambda+1.0000+c & 7.0000 \\
0.0000 & 7.0000 & \lambda+0.0385
\end{array}\right|=0
\end{aligned}
$$

i.e.

$$
\begin{aligned}
\lambda^{3}+ & (c+9.7685) \lambda^{2}+(8.7685 c-70.1454) \lambda \\
& +0.3361 c-428.5985=0 .
\end{aligned}
$$

According to Hurwitz's theorem, a necessary and sufficient condition for the negativity of the real parts of all the roots of the polynomial Eq. (13) is

$$
\left\{\begin{array}{l}
c+9.7685>0 \\
8.7685 c-70.1454>0 \\
0.3361 c-428.5985>0 \\
(c+9.7685)(8.7685 c-70.1454) \\
-(0.3361 c-428.5985)>0
\end{array}\right.
$$

by calculation, we have $c>1275.2112$.

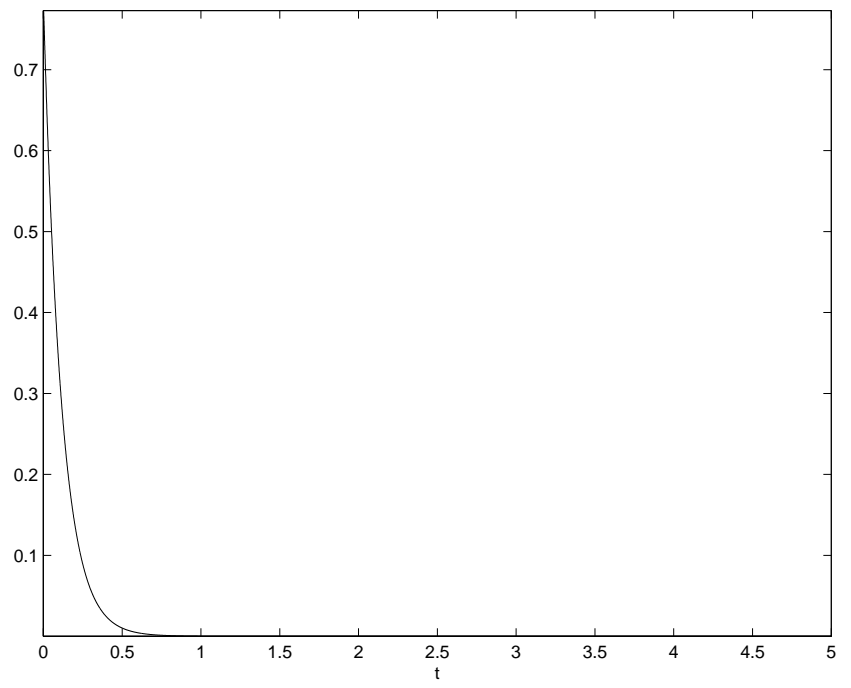

Fig. 1. Synchronization of two $x_{2}$-coupled Chua's circuits performed with $c=1301.0000$. 
By virtue of Corollary 1, system (7) and system (8) are indeed synchronized for any coupling constant $c>1275.2112$. Figure 1 shows the simulation result of the synchronization of (7) and (8) with $c=1300.0000$.

\section{Conclusion}

In this paper, we propose a new principle for synchronization of coupled chaotic oscillators by the Liapunov stability theory and Hurwitz Theorem. As an application example of this principle, we have proven the conjecture that synchronization of two coupled Chua's circuits with coupled second variable can be achieved provided that the coupling constant is enough large.

\section{Acknowledgments}

This work is supported by National Natural Science Foundation and Shanghai National Natural Science Foundation.

\section{References}

Chua, L. O., Itoh, M., Kocarev, L. \& Eckert, K. [1993] "Chaos synchronization in Chua's circuit," J. Circuits Syst. Comput. 3, 93-108.
Chua, L. O., Yang, T., Zhong, G. Q. \& Wu, C. W. [1996] "Adaptive synchronization of Chua's oscillators," Int. J. Bifurcation and Chaos 6, 189-201.

Kolumban, G., Kennedy, M. P. \& Chua, L. O. [1997] "The role of synchronization in digital communication using chaos - Part I: Fundamentals of digital communications," IEEE Trans. Circuits Syst. I 44, 927-936.

Ogorzalek, M. [1993] "Taming chaos - Part 1: Synchronization," IEEE Trans. Circuits Syst. I 40, 693-699.

Pecora, L. M. \& Caroll, T. L. [1990] "Synchronization in chaotic systems," Phys. Rev. Lett. 64, 821-824.

Pecora, L. M., Carroll, T. L., Johnson, G. A., Mar, D. J. \& Heagy, J. F. [1997] "Fundamentals of synchronization in chaotic systems, concepts, and applications," Chaos 7, 520-543.

Rama Mohana Rao, M. [1980] Ordinary Differential Equations Theory and Application (Affiliated EastWest Press Private Limited).

Wang, X. F., Wang, Z. Q. \& Chen, G. [1999] "A new criterion for synchronization of coupled chaotic oscillators with application to Chua's circuits," Int. J. Bifurcation and Chaos 6, 1169-1174.

Wu, C. W. \& Chua, L. O. [1994] "A unified framework for synchronization and control of dynamical systems," Int. J. Bifurcation and Chaos 4, 979-998. 